\title{
EMISSÕES SOLARES TIPO-U E TIPO-J DECIMÉTRICAS REGISTRADAS PELO BRAZILIAN SOLAR SPECTROSCOPE (BSS)
}

\section{SOLAR DECIMETER TYPE-U AND TYPE-J EMISSIONS RECORDED BY THE BRAZILIAN SOLAR SPECTROSCOPE (BSS)}

\author{
José Augusto S. S. Dutra ${ }^{1}$ \\ Francisco Carlos Rocha Fernandes ${ }^{2}$ \\ Rafael Douglas Cunha-Silva ${ }^{3}$
}

RESUMO: Neste trabalho são apresentados os resultados da análise de cinco emissões solares tipo- $U$ e tipo-J registradas pelo rádio espectrógrafo "Brazilian Solar Spectroscope" (BSS) em frequências decimétricas (1,0 - 2,5 $\mathrm{GHz}$ ), entre junho de 2000 e outubro de 2001. As características espectro-temporais das emissões foram medidas a partir dos espectros dinâmicos. Considerando a emissão de plasma no segundo harmônico, foram inferidos os seguintes parâmetros da fonte emissora: densidade de fluxo inferior a 20 - 80 u.f.s.; comprimento dos arcos magnéticos da ordem de $(0,3-5,1) \times 10^{10} \mathrm{~cm}$; velocidade dos feixes de elétrons de $(0,47-1,16) \times 10^{5}$ $\mathrm{km} / \mathrm{s}$; densidade máxima dos feixes de elétrons de $(0,56-1,95) \times 10^{5} \mathrm{~cm}^{-3}$; máximo de $(2,53-7,37) \times 10^{31}$ elétrons no feixe; temperatura do topo dos arcos magnéticos de $(2,5-15,5) \times 10^{6} \mathrm{~K}$; e limite inferior para a intensidade do campo magnético na região do plasma confinado de $7-22$ G. Os resultados são comparados com determinações publicadas na literatura.

Palavras-chave: Flares solares; emissão decimétrica; emissões tipo-U e tipo-J.

ABSTRACT: We present the results of the analysis of five type- $U$ and type-J bursts recorded by the Brazilian Solar Spectroscope (BSS) in the decimeter frequency range, between June 2000 and October 2001. The frequency-time characteristics for the bursts were determined from the dynamic spectra. Considering those emissions were generated by plasma emission mechanism, the following physical parameters were estimated: flux density less that $20-80$ s.f.u.; length of magnetic loops of $(0.3-5.1) \times 10^{10} \mathrm{~cm}$; electron beam velocity of $(0.47-1.16) \times 10^{5} \mathrm{~km} / \mathrm{s}$; maximum electron beam density of $(0.56-1.95) \times 10^{5} \mathrm{~cm}^{-3}$; upper limit of $(2.53-7.37)$ $\times 10^{31}$ electrons in the beam; coronal loop apex temperature of $(2.5-15.5) \times 10^{6} \mathrm{~K}$; and low limit for magnetic field in the region of confined plasma of $7-22 \mathrm{G}$. These results are compared with those reported previously in the literature.

Keywords: Solar flares; decimeter emission; type-U and type $\mathrm{J}$ bursts.

\footnotetext{
${ }^{1}$ Mestre em Física e Astronomia - Universidade do Vale do Paraíba - Univap e Professor Efetivo da Secretaria de Educação do Estado de São Paulo. E-mail: jassd09@hotmail.com.

Doutor em Astrofísica - Instituto Nacional de Pesquisas Espaciais - INPE / Space Sciences Laboratory - University Of California Berkeley e Professor Titular da Univap. E-mail: guga@univap.br.

${ }^{3}$ Doutorando em Física e Astronomia - Univap. E-mail: rafdouglas@univap.br.
} 


\section{INTRODUÇÃO}

Os dados de emissões solares em radiofrequências (métricas, decimétricas e milimétricas) são importantes para a investigação de problemas ainda em discussão na física solar, principalmente relacionados aos processos de armazenamento e liberação de energia, à aceleração e ao transporte de partículas aceleradas e à caracterização e localização da região de aceleração, como citado por Aschwanden (2005).

As radioemissões solares são importantes diagnósticos para investigações dos flares e outros fenômenos solares impulsivos, uma vez que a análise dessas emissões possibilita a determinação da densidade eletrônica da fonte emissora, quando o mecanismo é a emissão de plasma de feixes de elétrons acelerados durante flares solares (BENZ, 1996).

Com base nas suas características morfológicas presentes nos espectros dinâmicos, as radioemissões solares podem ser classificadas em emissões tipo I, II, III, IV e IV (MCLEAN, 1985).

As emissões tipo I apresentam banda estreita em frequência (cerca de $2,5 \%$ da frequência de observação) e curta duração (da ordem de 1 segundo). As emissões tipo II são observadas principalmente entre $0,1 \mathrm{e}$ $200 \mathrm{MHz}$ com largura de banda instantânea estreita (da ordem de $\mathrm{MHz}$ ) e caracterizadas geralmente por um par de emissões harmônicas com lenta taxa de deriva em frequência (inferior a $1 \mathrm{MHz} / \mathrm{s}$ ).

As emissões tipo IV são divididas em estacionárias (emissão contínua intensa de banda larga que sucede um grande flare solar) e não-estacionárias (fonte de emissão contínua em movimento - plasmóide denso ejetado durante um flare).

As emissões tipo $V$ são emissões contínuas, entre 10 e $100 \mathrm{MHz}$, com duração de cerca de 2 minutos, que ocorrem durante ou logo após a um grupo de emissões tipo III.
As emissões tipo III geralmente ocorrem em grupos (dezenas ou centenas de emissões individuais) com duração total de minutos e apresentam altas taxas de deriva em frequência (superior a 100 $\mathrm{MHz} / \mathrm{s}$ ). São geradas pelo mecanismo de emissão de plasma e estão diretamente associadas a feixes de elétrons energéticos propagando-se ao longo das linhas de campo magnético na atmosfera solar com velocidades sub-relativísticas $(0,1-0,3 \mathrm{c}$, sendo $c$ a velocidade da luz no vácuo).

Quando os feixes se deslocam em direção à alta coroa solar, ao longo das linhas abertas de campo magnético, geram emissões tipo III métricas, com taxa de deriva em frequência negativa. Quando o movimento dos feixes é em direção à fotosfera solar, ao longo das configurações magnéticas fechadas geram emissões tipo III decimétricas com taxa de deriva positiva (SAWANT; FERNANDES; NERI, 1994; MELÉNDEZ et al., 1999; CECATTO et al., 2003; MÉSZÁROSOVÁ et al., 2008).

$\begin{array}{lll}\begin{array}{c}\text { Diversas } \\ \text { apresentam }\end{array} & \begin{array}{c}\text { estruturas finas que } \\ \text { aspectos }\end{array} \text { morfológicos }\end{array}$
semelhantes às explosões tipo III, como as emissões tipo-U e tipo-J são, muitas vezes, designadas como variantes de tipo III ou simplesmente tipo III-like. Tais emissões também são geradas por emissão de plasma de elétrons acelerados e viajando ao longo das estruturas magnéticas fechadas (loops) na cromosfera e na baixa coroa solar (WILD; SMERD; WEISS, 1963; FOKKER, 1970; ASCHWANDEN; BENZ; MONTELLO, 1994).

Emissões tipo-U invertido (ou apenas tipo-U) apresentam taxa de deriva em frequência variando gradualmente de negativa (de alta para baixa frequência) para positiva, passando pela deriva nula, na chamada frequência de retorno ou inversão.

As emissões tipo-U são principalmente observadas em comprimentos de onda métricos e decamétricos (STONE; FAINBERG, 1971; KARLICKÝ; TLAMICHA, 1976). No entanto, emissões tipo-U decimétricas também foram relatadas (YAO 
et al., 1997a; 1997b; GÜDEL; BENZ, 1988; ASCHWANDEN et al., 1992; AURASS; KLEIN; MARTENS, 1994; AURASS; KLEIN, 1997; WANG et al., 2001; CECATTO et al., 2002; DUTRA; GURANIERI; FERNANDES, 2008; DUTRA, 2010; FERNANDES et al., 2012) até frequência de $3,8 \mathrm{GHz}$ (WANG et al., 2001).

Por sua vez, as estruturas finas denominadas tipo-J (ou $\mathrm{J}$ invertido) são variantes de emissão tipo-U. Apresentam interrupção da emissão no ramo descendente (perda de energia dos elétrons do feixe emissor antes de percorrer a porção descendente do arco magnético) (FOKKER, 1969). A Fig. 1 mostra uma relação entre a geometria da configuração magnética das regiões de aceleração e a morfologia das emissões tipo III, $U$ e $\mathrm{J}$ presentes nos espetros dinâmicos.
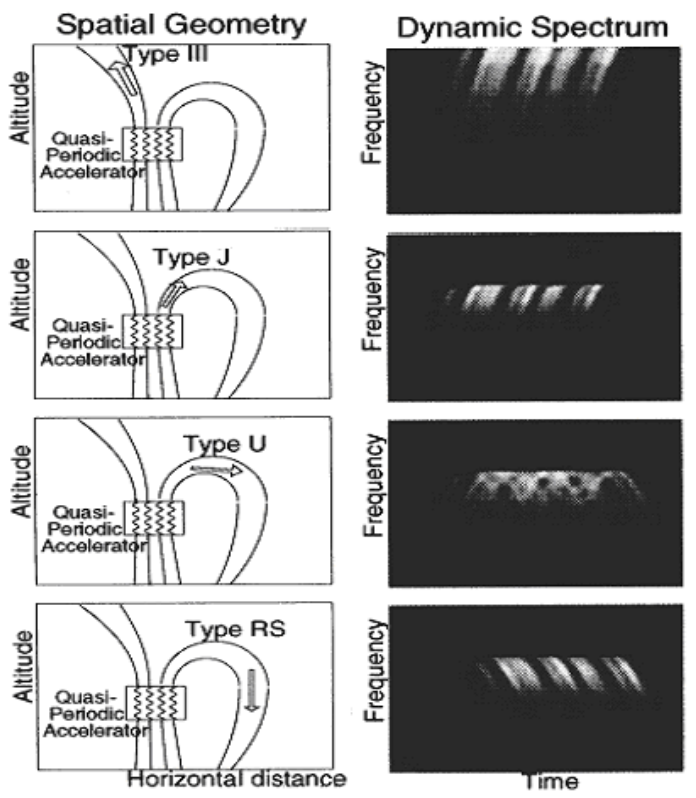

Fig. 1 - (Esquerda) Representação da configuração magnética de arcos nas regiões de aceleração. (Direita) Respectivos espectros dinâmicos das radioemissões geradas por feixes de elétrons acelerados nas linhas de campo magnético abertas ou fechadas. Fonte: Adaptada de Aschwanden, Benz e Montello (1994).

Pela análise de emissões tipo-U e tipo$\mathrm{J}$, é possível determinar aspectos da geometria dos arcos magnéticos e investigar características da fonte (ASCHWANDEN et al., 1992; YAO et al., 1997a; 1997b; WANG et al., 2001). Análise detalhada das emissões tipo-U, registradas pelo Brazilian Solar Spectroscope (BSS) (FERNANDES, 1997; SAWANT et al., 2001), foram relatadas por Dutra (2010) e Fernandes et al. (2012). O presente trabalho complementa as análises das 5 emissões tipo- $U$ e tipo-J apresentadas nesses dois trabalhos.

\section{METODOLOGIA EXPERIMENTAL}

A partir dos dados de explosões solares registradas pelo $B S S$ na faixa de frequências decimétricas $(1,0-2,5 \mathrm{GHz})$, entre 1999 e 2001 (FERNANDES, 2003a; 2003b; 2003c), foram selecionadas para análise cinco estruturas finas na radioemissão com morfologia tipo-U ou tipo$\mathrm{J}$ (mensurável dentro da faixa de frequência de observação), listadas na Tabela 1. 
Tabela 1 - Emissões tipo-U e tipo-J selecionadas para a análise e flares solares em raios- $X$ associados

\begin{tabular}{cccccc}
\hline$N^{\circ}$. & Data & $\begin{array}{c}\text { Tempo } \\
\text { de pico } \\
\text { (UT) }\end{array}$ & Tipo & $\begin{array}{c}\text { Flare em } \\
\text { raios-X }\end{array}$ & $\begin{array}{c}\text { Tempo de pico } \\
\text { em raios-X } \\
\text { (UT) }\end{array}$ \\
\hline 1 & $06 /$ jun/2000 & $16: 28: 00$ & $\mathrm{U}$ & $\mathrm{X} 2.3$ & $15: 25$ \\
2 & $12 / \mathrm{dez} / 2000$ & $14: 43: 14$ & $\mathrm{U}$ & $\mathrm{M} 1.3$ & $15: 07$ \\
3 & $07 / \mathrm{jun} / 2001$ & $16: 11: 38$ & $\mathrm{~J}$ & $\mathrm{C} 2.5$ & $16: 09$ \\
4 & $22 /$ ago/2001 & $15: 14: 22$ & $\mathrm{~J}$ & $\mathrm{C} 3.7$ & $15: 06$ \\
5 & $25 /$ out/2001 & $15: 13: 18$ & $\mathrm{U}$ & $\mathrm{X} 1.3$ & $15: 02$ \\
\hline
\end{tabular}

A Fig. 2 apresenta os espectros dinâmicos das cinco emissões analisadas e dos perfis de emissão em raios- $X$ moles.

A Tabela 1 também apresenta a classe e o tempo de pico dos flares solares em raios- $X$ associados. Nota-se que, exceto para a emissão tipo-U № 2, todas apresentam tempo de pico posterior ao tempo de pico do flare em raios- $X$ associado.

Durante a fase impulsiva, os elétrons presentes na coroa solar, próximos às regiões de reconexão magnética podem sofrer processos de aceleração em diferentes estágios (aceleração primária, secundária, etc), caracterizando feixes que viajam ao longo das estruturas magnéticas e produzem radioemissão devido à interação com o plasma, como as emissões tipo-U identificadas.

Em decorrência do tempo necessário para a desestabilização desses feixes e posterior conversão em radioemissões, essas ocorrem geralmente minutos após o início da fase impulsiva, próximo ao máximo de emissão em raios- $X$ moles, como registrado nas emissões tipo- $U$ e tipo- $J$ identificadas. A emissão № 2 ocorreu na fase impulsiva, cerca de 24 minutos antes do pico da emissão em raios-X. E os tempos de pico das emissões 3,4 e 5 , ocorreram, respectivamente, cerca de 2, 8 e 11 minutos após o instante de máximo do flare em raios- $X$ associado.

Apenas a emissão tipo-U № 1 apresentou um máximo cerca de 1 hora após o pico em raios- $X$ moles. Esse evento apresentou uma fase de decaimento de longa duração, durante a qual foram registradas diversas emissões características do aprisionamento de elétrons, o que, provavelmente, justifica a emissão ser observada em tempo posterior. 
(a) Emissão tipo U

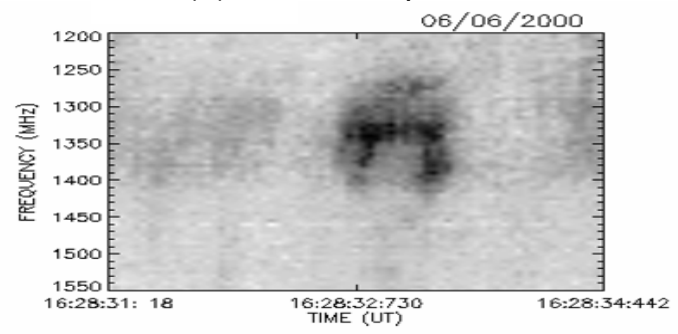

(b) Emissão tipo U

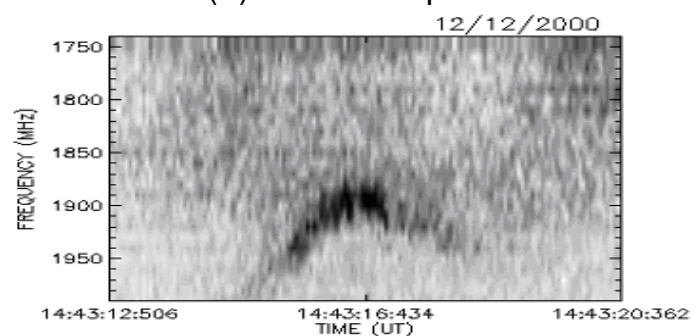

(c) Emissão tipo J

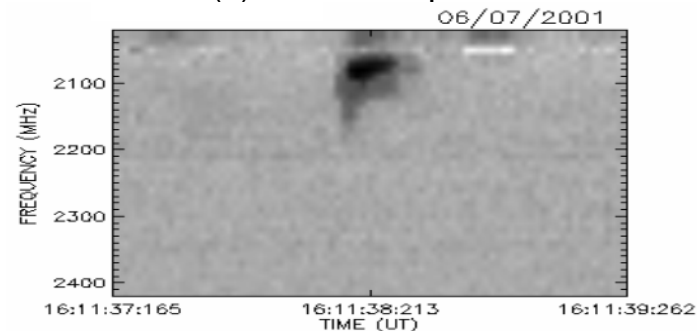

(d) Emissão tipo J

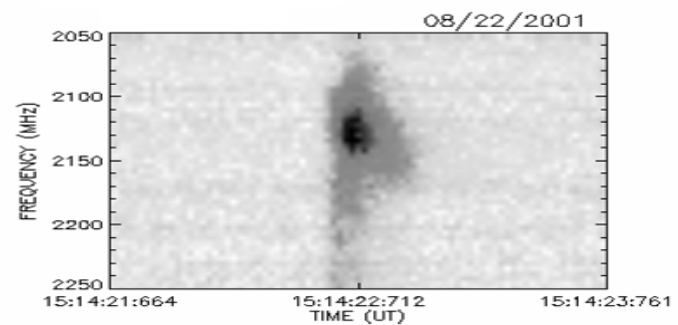

(e) Emissão tipo U

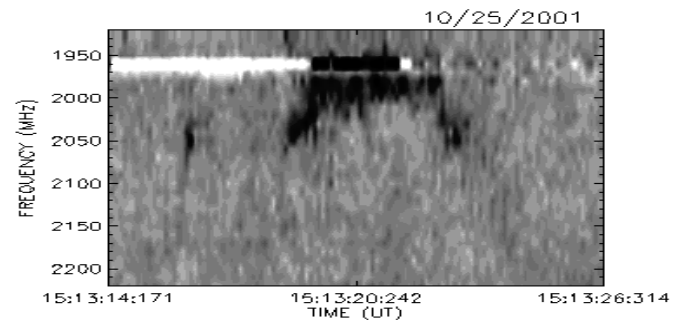

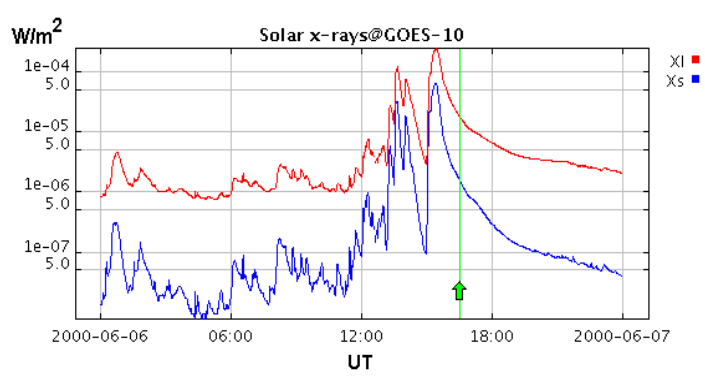
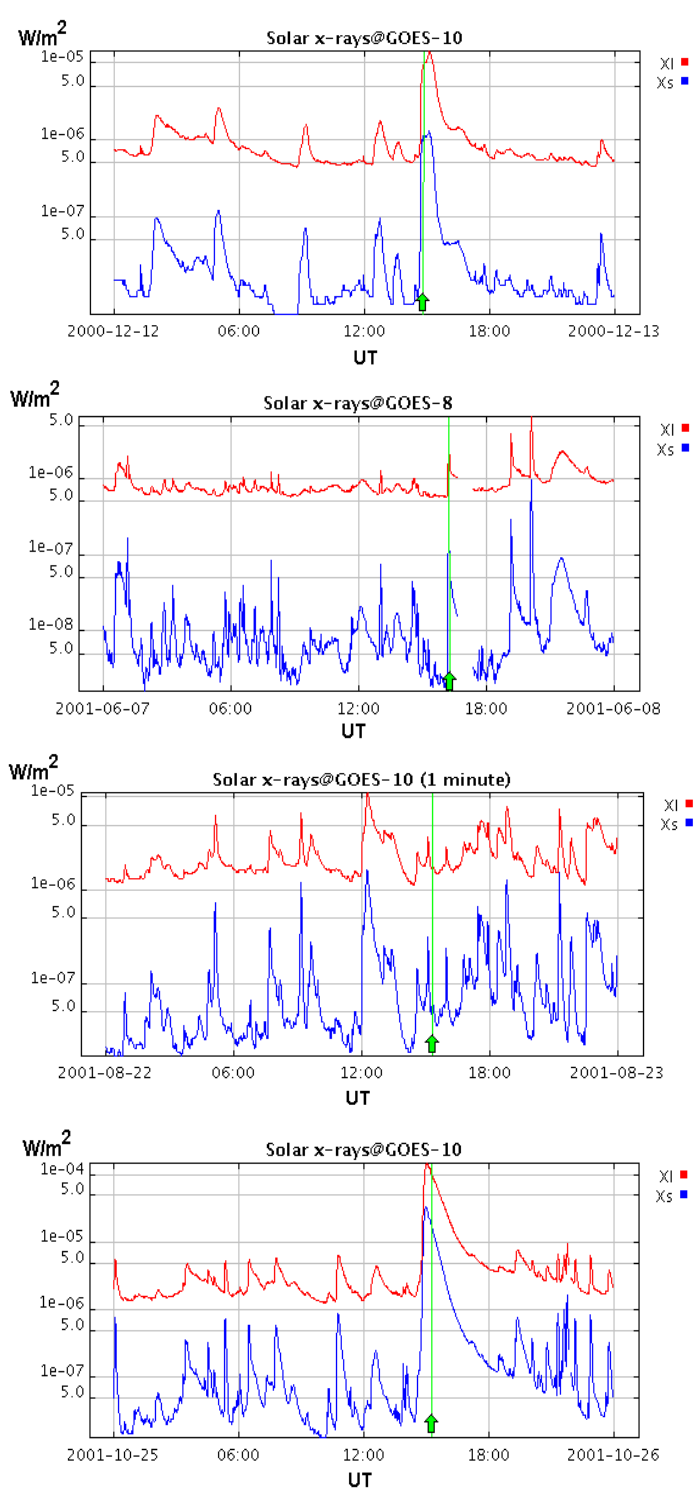

Fig. 2 - A coluna da esquerda mostra os espectros dinâmicos das emissões tipo-U e tipo-J registradas em: (a) 06 de junho de 2000 ( 16:28 UT); (b) 12 de dezembro de 2000 ( 14:43 UT); (c) 07 de junho de 2001 ( 16:11 UT); (d) 22 de agosto de 2001 ( 15:14 UT) e (e) 25 de outubro de 2001 ( 15:13 UT). A coluna da direita apresenta os perfis em raios-X mole do satélite GOES, mostrando os flares associados às emissões tipo-U e tipo-J selecionadas (seta verde). 


\section{RESULTADOS}

\subsection{Parâmetros observacionais}

Os dados de rádio emissões solares são registrados pelo BSS em unidades arbitrárias de intensidade. A estimativa da intensidade (densidade de fluxo) de pico das emissões em unidades de fluxo solar (u.f.s.) (1 u.f.s. $=10^{-22} \mathrm{~W} \mathrm{~Hz}^{-1} \mathrm{~m}^{-2}$ ) foi feita por meio de um procedimento de calibração, proposto por Guidice e Castelli (1971) e aplicado por Fernandes (1992) para dados do BSS, baseado na comparação entre o nível de intensidade do pico da emissão com o nível do Sol calmo (background) e um nível padrão gerado instrumentalmente.

A calibração desse nível foi feita pela interpolação de intensidade de fluxo em várias frequências obtida do Solar Geophysical Data (SGD).

Para a interpolação, foram utilizadas as frequências $610 \mathrm{MHz}$ e $1415 \mathrm{MHz}$ para a emissão № 1 e $1415 \mathrm{MHz}$ e $2695 \mathrm{MHz}$ para as demais emissões analisadas. Os valores máximos de densidade de fluxo $(S)$ das emissões são mostrados na Tabela 2.

Dos espectros dinâmicos das emissões selecionadas foram obtidos os seguintes parâmetros observacionais: tempo de início $\left(t_{i}\right)$; tempo de fim $\left(t_{f}\right)$; duração total
$(\Delta t)$; intervalo de tempo do ramo ascendente $\left(\Delta t_{a}\right)$; intervalo de tempo do ramo descendente $\left(\Delta t_{d}\right)$; frequência de início $\left(f_{i}\right)$; frequência de fim $\left(f_{f}\right)$; frequência de retorno $\left(f_{r}\right)$; banda total de frequência $(\Delta f)$; banda de frequência do ramo ascendente $\left(\Delta f_{a}\right)$; banda de frequência do ramo descendente $\left(\Delta f_{d}\right)$; e largura de banda instantânea $\left(\Delta f_{i}\right)$.

A metade do intervalo de frequência entre dois canais sucessivos foi tomada como erro de cada medida de frequência e a metade do intervalo entre duas varreduras sucessivas, o erro para as medidas de tempo.

A duração total $(\Delta t)$ para uma explosão foi determinada diretamente da diferença entre os tempos de fim $\left(t_{f}\right)$ e de início $\left(t_{i}\right)$ medidos, $\Delta t=t_{f}-t_{i}$. Analogamente, a banda total de frequência $(\Delta f)$ foi determinada como o maior intervalo de frequência entre a frequência extrema (inicial ou final) e a frequência de retorno $\left(\mathrm{f}_{\mathrm{r}}\right)$, ou seja, $\Delta f=f_{i}-f_{r}$, se $f_{i}>f_{f}$ e $\Delta f=f_{f}-f_{r}$ se $f_{i}<f_{f}$.

A Tabela 2 apresenta as características espectro-temporais determinadas para as cinco emissões analisadas. Os procedimentos de análise são descritos, em detalhes, por Dutra (2010).

Tabela 2 - Parâmetros observados das emissões tipo-U e tipo-J selecionadas: Densidade de fluxo do pico $(S)$; frequência de início $\left(f_{i}\right)$; frequência de fim $\left(f_{f}\right)$; frequência de retorno $\left(f_{r}\right)$; Banda total de frequência $(\Delta t)$ e Duração total $(\Delta t)$

\begin{tabular}{ccccccc}
\hline & $\begin{array}{c}\boldsymbol{S} \\
\mathbf{N}^{\circ} .\end{array}$ & $\begin{array}{c}\boldsymbol{f}_{\boldsymbol{i}} \\
\text { (u.f.s.) }\end{array}$ & $\begin{array}{c}\boldsymbol{f}_{\boldsymbol{f}} \\
(\mathbf{M H z})\end{array}$ & $\begin{array}{c}\boldsymbol{f}_{\boldsymbol{r}} \\
(\mathbf{M H z})\end{array}$ & $\begin{array}{c}\Delta \boldsymbol{f} \\
(\mathbf{M H z})\end{array}$ & $\begin{array}{c}\Delta \boldsymbol{t} \\
(\mathbf{m s})\end{array}$ \\
\hline 1 & $<20$ & $1420 \pm 3$ & $1255 \pm 3$ & $1338 \pm 3$ & $165 \pm 3$ & $650 \pm 25$ \\
2 & $<30$ & $1970 \pm 5$ & $1870 \pm 5$ & $1920 \pm 5$ & $100 \pm 5$ & $3400 \pm 25$ \\
3 & $<80$ & $2170 \pm 5$ & $2050 \pm 5$ & $2110 \pm 5$ & $120 \pm 5$ & $510 \pm 10$ \\
4 & $<40$ & $2050 \pm 3$ & $2246 \pm 3$ & $2148 \pm 3$ & $196 \pm 3$ & $550 \pm 10$ \\
5 & $<25$ & $2060 \pm 5$ & $2040 \pm 5$ & $1940 \pm 5$ & $120 \pm 5$ & $4400 \pm 50$ \\
\hline
\end{tabular}

Para as emissões tipo-U, apresentando os ramos ascendente e descendente, a determinação das taxas de deriva em frequência de cada um dos ramos foi obtida a partir de um ajuste linear entre os picos de emissão identificados nos extremos de cada ramo. Assim, foram obtidas as taxas de deriva em frequência dos ramos ascendente e descendente de cada uma das emissões tipo-U analisadas, 
considerando as frequências de início, de retorno e de fim das emissões e seus respectivos tempos.

A Tabela 3 apresenta as taxas de deriva em frequência dos ramos ascendente e descendente determinadas.

Yao et al. (1997a; 1997b) obtiveram taxa de deriva em frequência para os ramos ascendente e descendente de emissões tipo-U de $1250 \mathrm{MHz} / \mathrm{s}$ e $2250 \mathrm{MHz} / \mathrm{s}$, respectivamente. Wang et al. (2001) obtiveram 1730 - $3000 \mathrm{MHz} / \mathrm{s}$ e 2010 - 1700 $\mathrm{MHz} / \mathrm{s}$, para os ramos ascendente $\mathrm{e}$ descendente, respectivamente.

Valores superiores, no intervalo de $3600-4500 \mathrm{MHz} / \mathrm{s}$, foram obtidos por Ning et al. (2003), para uma emissão tipo-N parcial observada em $6 \mathrm{GHz}$.

\section{Tabela 3 - Taxa de deriva em frequência dos ramos ascendente e descendente das emissões tipo-U e tipo-J analisadas}

\begin{tabular}{ccc}
\hline & \multicolumn{2}{c}{$\begin{array}{c}\text { Taxa de deriva em frequência } \\
\text { (MHz/s) }\end{array}$} \\
\cline { 2 - 3 } № & $\begin{array}{c}\text { Ramo } \\
\text { ascendente }\end{array}$ & $\begin{array}{c}\text { Ramo } \\
\text { descendente }\end{array}$ \\
\hline 1 & 275 & 1160 \\
2 & 49 & 74 \\
3 & 61 & 83 \\
4 & - & - \\
5 & 47 & 60 \\
\hline
\end{tabular}

Os valores obtidos, da ordem de dezenas de $\mathrm{MHz} / \mathrm{s}$, são cerca de uma ordem de grandeza inferior aos valores típicos. A justificativa é que neste trabalho não foram considerados, na determinação das taxas de deriva, efeitos de projeção, sendo os arcos considerados sempre perpendiculares ao disco solar.

\subsection{Parâmetros físicos da fonte}

A partir dos parâmetros observacionais obtidos dos espectros dinâmicos, para cada explosão selecionada, foram estimados os principais parâmetros da emissão e da fonte emissora: a densidade eletrônica na fonte, a velocidade, a densidade e o número total de elétrons do feixe (agente excitador), a temperatura da região e o limite inferior para a intensidade do campo magnético na fonte.

A metodologia de determinação dos parâmetros físicos da fonte emissora das emissões tipo-U e tipo-J, descrita em detalhes por Dutra (2010), foi baseada nos trabalhos de Aschwanden et al. (1992), Yao et al. (1997a), Wang et al., (2001) e Ning et al. (2003). Os resultados são apresentados e discutidos a seguir.

\subsubsection{Densidade eletrônica}

No mecanismo de emissão de plasma, a frequência da rádio-emissão detectada pode ser considerada como a frequência de plasma $\left(f_{p}\right)$, que está relacionada com a densidade eletrônica $\left(N_{e}\right)$ da atmosfera solar pela equação (McLEAN, 1985, p.93):

$$
f_{p}=s 8,98 \times 10^{-3} \sqrt{N_{e}}(\mathrm{MHz}),
$$

sendo $s$ o parâmetro harmônico $(s=1$ para emissão no fundamental e $s=2$ no segundo harmônico).

Considerando que uma emissão tipo-U é gerada pelo mecanismo de emissão de plasma, a frequência de rádio-emissão observada será a própria frequência de plasma. De tal forma que a densidade eletrônica pode ser determinada. Para essa determinação, foi considerada a frequência média da banda total de frequência da rádioemissão, entre as frequências extremas da banda, ou seja, $f_{p}=\left(f_{i}+f_{r}\right) / 2$ e a emissão no segundo harmônico, isto é, considerando $s=2$.

\subsubsection{Velocidade do feixe de elétrons}

$\mathrm{Na}$ região da atmosfera solar associada às emissões de plasma na faixa decimétrica, os feixes de elétrons acelerados durantes os flares se propagam através de um meio relativamente denso, 
podendo ser rapidamente termalizados por colisões coulombianas. O tempo de colisão $\left(t_{D}\right)$, dado em segundos, pode ser obtido por (BOYD; SANDERSON, 1969):

$$
t_{D}=3,1 \times 10^{-20} \quad v_{b}^{3} N_{e}^{-1},
$$

sendo $N_{e}$ a densidade eletrônica e $v_{b}$ a velocidade do feixe de elétrons.

Dessa forma, considerando o tempo de colisão como sendo o tempo de duração total da emissão, foi obtida a velocidade mínima de propagação do feixe de elétrons. Os valores obtidos estão entre 0,16 e 0,50 c.

Yao et al. (1997a; 1997b) e Aschwanden et al. (1992) obtiveram velocidades da ordem de $(0,26-0,38) \mathrm{c}$ e $(0,14-0,21) \mathrm{c}$, respectivamente, nas faixas de frequência de $(1,1-1,7) \mathrm{GHz}$ e $(1,0$ - 2,8) $\mathrm{GHz}$. Ning et al. (2003) estimaram a velocidade do feixe emissor para a explosão tipo-N parcial em 0,33 c.

\subsubsection{Comprimento do arco}

A determinação do comprimento do arco magnético foi baseada em considerações cinemáticas (como sendo o produto da velocidade do feixe de elétrons pelo tempo de duração da rádio-emissão),

$$
L \approx t_{D} v_{b} .
$$

Os valores obtidos são da ordem de 3 $\times 10^{9} \mathrm{~cm}$, cerca de uma ordem de grandeza menor que os obtidos por Aschwanden et al. (1992) $\left(1,7 \times 10^{10} \mathrm{~cm}\right)$ a partir das observações com o VLA, pois não foram considerados efeitos de projeção ou inclinação do arco magnético em relação à linha visada.

\subsubsection{Temperatura da região emissora}

Rosner, Tucker e Viana (1978) apresentam uma relação entre a temperatura $(T)$, a pressão $(p)$ e o tamanho $(L)$ da estrutura magnética em forma de arco:

$$
T=1,4 \times 10^{3}(p L)^{1 / 3} .
$$

A lei dos gases ideais, $p=N_{e} k_{b} T$, sendo $k_{b}$ a constante de Boltzsmann, também é aplicável no caso do plasma da atmosfera solar. Assim, a temperatura da região emissora foi obtida a partir das densidades eletrônicas e dos comprimentos do arco:

$$
T=6,2 \times 10^{-4}\left(N_{e} L\right)^{1 / 2} .
$$

Os valores inferidos estão no intervalo de $2,6 \times 10^{6} \mathrm{~K}$ a $1,5 \times 10^{7} \mathrm{~K}$ e concordam com as determinações de Aschwanden et al., (1992), Yao et al. (1997a; 1997b) e Wang et al. (2001), que obtiveram temperaturas de $(2-8) \times 10^{6} \mathrm{~K}$. Estão também em acordo com os resultados de Fontenla et al. (1991), que mostram que componentes de diferentes temperaturas (entre $10^{4}$ e $10^{7} \mathrm{~K}$ ) coexistem num mesmo arco ativo.

3.2.6 Densidade e número total de elétrons do feixe

Como discutido anteriormente, a emissão tipo-U é gerada pelo mecanismo de emissão de plasma por feixes de elétrons acelerados, o mesmo mecanismo das emissões tipo III.

Segundo Fürst, Benz e Hirth (1982), a densidade de fluxo das emissões em ondas decimétricas geradas pelo mecanismo de emissão de plasma pode ser inferida por

$$
S=a\left(\frac{v_{I I I}}{c}\right)\left(\frac{f_{p}}{\Delta w}\right)\left(\frac{N_{b}}{N_{e}}\right)^{4}\left(\frac{v_{I I I}}{\Delta v}\right) V_{d m} N_{e} k T \boldsymbol{\delta}(6)
$$

em que $a=1,16 \times 10^{-5} ; v_{I I /} / c$ é a razão entre a velocidade do feixe estimada para o ramo ascendente das emissões tipo-U e a velocidade da luz no vácuo; $f_{p}$ é a frequência de plasma para a emissão no segundo harmônico $(\mathrm{Hz}) ; \Delta w$ é o espalhamento em frequência $(\mathrm{Hz}) ; N_{b}$ é a densidade do feixe de elétrons $\left(\mathrm{cm}^{-3}\right) ; N_{e}$ é a densidade eletrônica da região emissora $\left(\mathrm{cm}^{-3}\right) ; \Delta v$ é o espalhamento em velocidade do feixe de elétrons $(\mathrm{cm} / \mathrm{s}) ; V_{d m}$ é o volume da região de 
emissão $\left(\mathrm{cm}^{3}\right) ; \quad k$ é a constante de Boltzmann $\left(1,38 \times 10^{-16} \mathrm{erg} / \mathrm{K}\right) ; \quad T$ é a temperatura da fonte $(\mathrm{K})$ e $\delta$ é o parâmetro de absorção da radiação decimétrica.

Segundo Aschwanden, Benz e Schwartz (1983), o tamanho aparente $(R)$ da fonte em função da frequência de observação em $\mathrm{GHz}$, pode ser obtido por

$$
R(f)=15.000 \mathrm{f}_{\mathrm{GHz}}{ }^{-1,08} \mathrm{~km}
$$

Considerando a frequência média entre as frequências de início e de fim de cada emissão analisada (Tabela 2), foi obtido o tamanho da fonte e estimado o volume da região emissora. Os valores de volume obtidos estão no intervalo de $(0,28$ $1,31) \times 10^{27} \mathrm{~cm}^{3}$.

Então, substituindo, na equação (6), os valores dos parâmetros de plasma para a emissão decimétrica fornecidos por Fürst, Benz e Hirth (1982): $\left(v_{\| I} / \Delta v\right)=1 ;\left(f_{p} / \Delta w\right)=4$ e $\delta=0,1$ e os parâmetros da fonte determinados a partir da análise das emissões tipo-U e tipo-J, foi possível obter uma relação entre a densidade de fluxo da emissão e a densidade do feixe de elétrons emissor.

Assim, tomando a densidade de fluxo do pico máxima de cada emissão (2 $2^{\mathrm{a}}$. coluna da Tabela 2), foi estimada a densidade máxima do feixe de elétrons, que escapa da região fonte de emissão e viaja ao longo do arco magnético gerando, pela interação com - plasma confinado, as emissões decimétricas analisadas. Os valores de densidade inferidos estão no intervalo $5,61 \times$ $10^{4} \mathrm{~cm}^{-3}$ a $1,95 \times 10^{5} \mathrm{~cm}^{-3}$.

Considerando que tais valores representam o limite superior para a densidade dos feixes de elétrons, eles concordam com resultados obtidos por Fürst, Benz e Hirth (1982) de $5 \times 10^{4} \mathrm{~cm}^{-3}$, para emissões entre 600 e $1000 \mathrm{MHz}$ e por Melendez-Moreno (1997) de $6 \times 10^{4} \mathrm{~cm}^{-3} \mathrm{e}$
Fernandes (1992) de $7 \times 10^{4} \mathrm{~cm}^{-3}$ para emissões em $1600 \mathrm{MHz}$.

Considerando o volume da fonte emissora, o número máximo de elétrons do feixe $(n)$ necessários para gerar a emissão decimétrica pelo mecanismo de emissão de plasma obtido foi de $(2,53-7,37) \times 10^{31}$ elétrons.

Tais valores também estão de acordo com as determinações de Fürst, Benz e Hirth (1982), Melendez-Moreno (1997) e Fernandes (1992), que obtiveram $5 \times 10^{31}$ elétrons, $6 \times 10^{31}$ elétrons e $7 \times 10^{31}$ elétrons, respectivamente.

\subsubsection{Campo magnético}

O parâmetro $\beta$ expressa a razão entre a pressão térmica e a pressão magnética:

$$
\beta=3,47 \times 10^{-15}\left(\mathrm{Ne} T B^{-2}\right),
$$

sendo $B$ a intensidade do campo magnético, em Gauss. A condição para o plasma estar magneticamente confinado, como ocorre nos arcos magnéticos na coroa solar, é que a pressão magnética domine sobre a pressão do gás, ou seja, $\beta<1$.

Para as emissões tipo-U, o plasma coronal deve estar confinado na estrutura magnética. Assim, considerando $\beta<1$ na equação (8) e a densidade eletrônica e temperatura estimadas para as emissões analisadas, o limite inferior do campo magnético $\left(B_{\min }\right)$ foi estimado. Os valores obtidos foram de 10 - $20 \mathrm{G}$.

Yao et al. (1997b), Aschwanden et al. (1992) e Wang et al. (2001) obtiveram, respectivamente, 9,2 G, $13 \mathrm{G}$ e $21 \mathrm{G}$, como limite inferior do campo magnético no topo do arco.

Os parâmetros físicos determinados para as emissões tipo- $U$ e tipo-J são apresentados na Tabela 4. 
Tabela 4 - Parâmetros físicos determinados para as emissões tipo-U e tipo-J: densidade eletrônica $\left(N_{e}\right)$, velocidade do feixe de elétrons $\left(v_{b}\right)$, densidade do feixe de elétrons $\left(N_{b}\right)$, número total de elétrons no feixe $(n)$, comprimento do arco magnético

$(L)$, temperatura da região emissora $(T)$ e campo magnético $(B)$

\begin{tabular}{|c|c|c|c|c|c|c|c|}
\hline № & $\begin{array}{c}N_{e} \\
\left(10^{10} \mathrm{~cm}^{-3}\right)\end{array}$ & $\begin{array}{c}V_{b} \\
\left(10^{5} \mathrm{~km} / \mathrm{s}\right)\end{array}$ & $\begin{array}{c}N_{b} \\
\left(10^{5} \mathrm{~cm}^{-3}\right)\end{array}$ & $\begin{array}{c}n \\
\left(10^{31} \text { elé. }\right)\end{array}$ & $\begin{array}{c}L \\
\left(10^{10} \mathrm{~cm}\right)\end{array}$ & $\begin{array}{c}T \\
\left(10^{7} \mathrm{~K}\right)\end{array}$ & $\begin{array}{c}B \\
(\mathrm{G})\end{array}$ \\
\hline 1 & 0,55 & 0,47 & 0,56 & 7,37 & 0,3 & 0,26 & $>11$ \\
\hline 2 & 1,14 & 1,04 & 0,79 & 3,25 & 3,5 & 1,20 & $>18$ \\
\hline 3 & 1,37 & 0,59 & 1,95 & 5,85 & 3,0 & 0,40 & $>7$ \\
\hline 4 & 1,42 & 0,61 & 1,65 & 4,69 & 0,3 & 0,43 & $>22$ \\
\hline 5 & 1,23 & 1,16 & 0,71 & 2,53 & 5,1 & 1,50 & $>20$ \\
\hline
\end{tabular}

\section{CONCLUSÃO}

Neste trabalho, foram apresentados os resultados da análise de rádio-emissões decimétricas solares registradas pelo BSS classificadas como emissões tipo-U e tipo-J.

A morfologia das rádio-emissões nos espectros dinâmicos suportam as hipóteses que são geradas por mecanismo de emissão de plasma, ou seja, a partir da interação de feixes de elétrons acelerados, propagandose ao longo das estruturas magnéticas solares, com o plasma da atmosfera solar confinado nessas estruturas.

As características espectro-temporais de cada uma das cinco estruturas finas com morfologia tipo-U ou tipo-J presentes na rádio-emissão solar decimétrica (950 - 2100 $\mathrm{MHz}$ ), observadas pelo BSS entre junho de 2000 e outubro de 2001.

A partir das medidas obtidas, foram determinados os principais parâmetros do agente e da fonte emissora. Os resultados mostram os seguintes intervalos de valores para os parâmetros determinados:

a) densidade de fluxo: $>20-80$ u.f.s.; b) densidade eletrônica: $(0,55-1,42) \times$ $10^{10} \mathrm{~cm}^{-3}$;

c) comprimento do arco: $(0,3-5,1) \times 10^{10}$ $\mathrm{cm}$;

d) velocidade do feixe: $(0,16-0,53) \mathrm{c}$;

e) densidade do feixe: $(0,56-1,95) \times 10^{5}$ $\mathrm{cm}^{-3}$;

f) número total de elétrons do feixe: (2,53 $7,37) \times 10^{31}$ elétrons;

g) temperatura do topo do arco magnético: $(0,26-1,50) \times 10^{7} \mathrm{~K} ; \mathrm{e}$

h) limite inferior para a intensidade do campo magnético: $(7-22) \mathrm{G}$.

Os valores obtidos estão de acordo com determinações relatadas na literatura para emissões tipo-U e suas variantes, como tipo-J e tipo-N, observadas na faixa decimétrica $(1,0-6,0 \mathrm{GHz})$, como mostra a Tabela 5. 
Tabela 5 - Comparação dos resultados obtidos da análise das emissões tipo-U e tipo$J$, para a densidade de fluxo (S), a velocidade do feixe de elétrons $\left(v_{b}\right)$, a temperatura do topo da região emissora $(T)$ e o limite inferior para a intensidade do campo magnético $\left(B_{\min }\right)$, com resultados de trabalhos publicados na literatura

\begin{tabular}{|c|c|c|c|c|c|}
\hline $\begin{array}{c}f \\
\mathrm{GHz}\end{array}$ & $\begin{array}{c}S \\
\text { (u.f.s.) }\end{array}$ & $\begin{array}{l}V_{b} \\
(\mathbf{c})\end{array}$ & $\begin{array}{c}T \\
\left(\times 10^{6} \mathrm{~K}\right)\end{array}$ & $\begin{array}{l}B_{\min } \\
(\mathbf{G})\end{array}$ & Referência \\
\hline $1,4-2,2$ & $20-80$ & $0,16-0,53$ & $2,6-15,5$ & $7-22$ & Este trabalho \\
\hline $1,1-1,7$ & $2-6$ & $0,26-0,38$ & 8,0 & 13 & Aschwanden et al. (1992) \\
\hline $1,0-2,8$ & $100-150$ & $0,14-0,21$ & 6,3 & 9,2 & Yao et al. (1997a; 1997b) \\
\hline $2,6-3,8$ & $140-200$ & - & 2,0 & 21 & Wang et al. (2001) \\
\hline $5,2-6,2$ & - & 0,33 & 10,0 & 130 & Ning et al. (2003) \\
\hline
\end{tabular}

A Fig. 3 mostra um cenário esquemático de uma região ativa com a presença de arcos magnéticos em interação, na qual são identificados os valores correspondentes dos parâmetros determinados no presente trabalho para a região emissora e para os feixes de elétrons acelerados, responsáveis pelas emissões tipo-U e tipo-J.

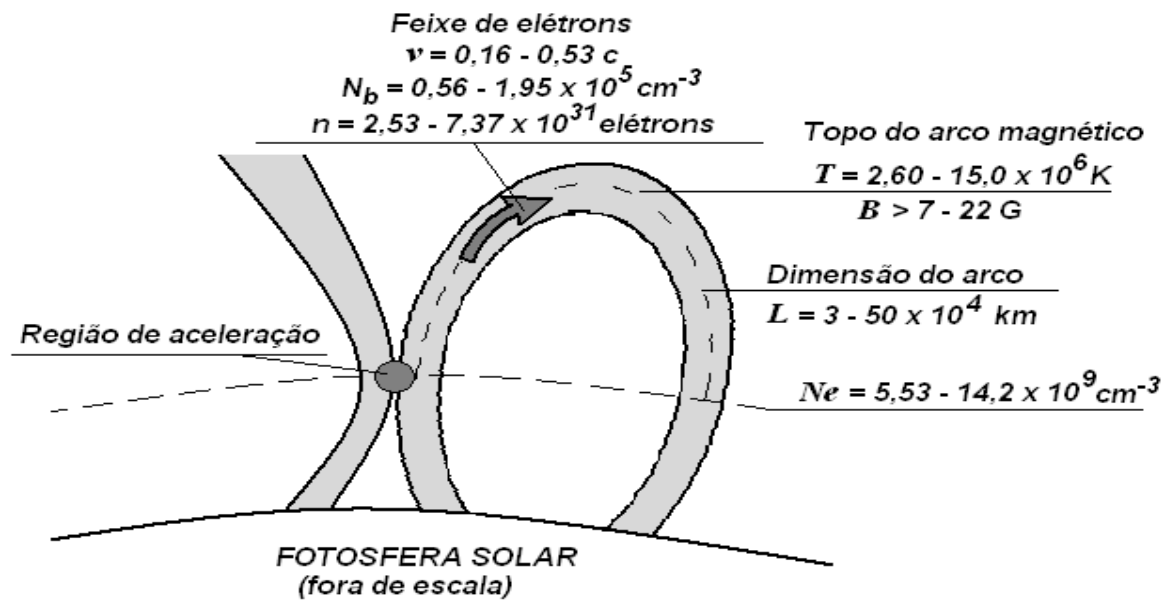

Fig. 3 - Representação esquemática (fora de escala) de uma região ativa com a presença de arcos magnéticos em interação. São identificados os intervalos de valores dos parâmetros determinados a partir da análise das emissões tipo-U e tipo-J.

Fonte: Adaptado de Dutra (2010).

\section{AGRADECIMENTOS}

J. A. S. S. Dutra e R. D. Cunha-Silva agradecem à Capes e à FVE/Univap pelas bolsas de Mestrado. R. D. Cunha-Silva agradece à FAPESP (Proc. 2012/00009-5) pela bolsa de Doutorado. As análises das emissões solares foram realizadas com apoio do CNPq (Proc. 480045/2008-9 Edital Universal). Os autores agradecem ao
Grupo de Física do Meio Interplanetário (FMI) da Divisão de Astrofísica (DAS) do Instituto Nacional de Pesquisas Espaciais (INPE), pelos dados das emissões solares analisados neste trabalho. Os autores agradecem, também, aos revisores pelas sugestões e comentários, que contribuíram para melhorar o texto final do presente trabalho. 


\section{REFERÊNCIAS}

ASCHWANDEN, M. J. et al. Decimetric solar type bursts: VLA and PHOENIX observations. Astrophysical Journal, v. 391, p. 380-392, 1992.

ASCHWANDEN, M. J.; BENZ, A. O.; SCHWARTZ, R. A. The timing of electron beam signatures in hard $\mathrm{X}$-ray and radio: solar flare observations by BATSE/Compton Gamma Ray Observatory and Phoenix. Astrophysical Journal, v. 417, p. 790-804, 1993.

ASCHWANDEN, M. J.; BENZ, A. O.; MONTELLO, M. L. Coherent-phase or random-phase acceleration of electron beams in solar flares. Astrophysical Journal, v. 431, n. 1, p. 432-449, 1994.

ASCHWANDEN, M. J. Physics of the Solar Corona: an introduction with problems and solutions. 2nd ed. Chichester, UK: Springer and Praxis Publishing Ltd., 2005.

AURASS, H.; KLEIN, K.-L.; MARTENS, P. C. $\mathrm{H}$. First detection of correlated electron beams and plasma jets in radio and soft $x$ ray data. Solar Physics, v. 155, n. 1, p. 203206, 1994.

AURASS, H.; KLEIN, K.-L. Spectrographic and imaging observations of solar type $U$ radio bursts. Astronomy and Astrophysics Supplement Series, v. 123, p. 279-304, 1997.

BENZ, A. O. Radio Astronomical Diagnostics. Lecture Notes in Physics, v. 468, p. 213, 1996.

BOYD, T. J. M.; SANDERSON, J. J. Plasma Dynamics, London: Nelson, 1969. (Applications of mathematics series).

CECATTO, J. R. et al. Decimetric Reverse Drift and U-Type Bursts in the April 9, 2001 Flare. COSPAR Colloquia Series, v. 13, p. 313, 2002.

CECATTO, J. R. et al. High resolution time profile of decimetric type-III bursts. Advances in Space Research, v. 32, n. 12, p. 25332537, 2003.
DUTRA, J. A. S. S.; GURANIERI, F. L.; FERNANDES, F. C. R. Estruturas finas presentes na radioemissão solar em ondas decimétricas: parâmetros e caracterização da região de aceleração. Anais do VIII EPG - Encontro Latino-Americano de PósGraduação, UNIVAP, 2008.

DUTRA, J. A. S. S. Estruturas finas tipo U e tipo $\mathrm{J}$ presentes na rádio-emissão solar em ondas decimétricas. São José dos Campos, SP, 2010. Dissertação (Mestrado em Física e Astronomia) - Instituto de Pesquisa e Desenvolvimento, Universidade do Vale do Paraíba, São José dos Campos, 2010.

FERNANDES, F. C. R. Espectrógrafo Decimétrico de alta sensibilidade e resolução: análise preliminar das explosões solares. Dissertação (Mestrado em Ciência Espacial) INPE, São José dos Campos. INPE-5537-TDI/525, 1992.

Espectrógrafo Digital Decimétrico de banda larga e investigações de flares solares em rádio e raios- $X$. Tese (Doutorado em Astrofísica) INPE, São José dos Campos. 1997. (INPE-6396-TDI/612)

Catálogo de espectros dinâmicos de explosões solares decimétricas registradas pelo Brazilian Solar Spectroscope (BSS): 1999. INPE-9654RPQ/740, INPE, 2003a.

Catálogo de espectros dinâmicos de explosões solares decimétricas registradas pelo Brazilian Solar Spectroscope (BSS): 2000. INPE-9653RPQ/739, INPE, 2003b.

Catálogo de espectros dinâmicos de explosões solares decimétricas registradas pelo Brazilian Solar Spectroscope (BSS): 2001. INPE-9652RPQ/738, INPE, 2003c.

FERNANDES, F. C. R. et al. Flaring loop parameters estimated from solar decimeter type U-like and type J-like fine structures. Advances in Space Research, v. 49, p. 1607-1614, 2012. 
FOKKER, A. D. Spectral Characteristics of Medium-Sized Solar Radio Events. Solar Physics, v. 8, n. 2, p. 376-387, 1969.

. Trajectories Followed by U-Like Solar Radio Bursts. Solar Physics, v. 11, n. 1, p. 92-103, 1970.

FONTENLA, J. M. et al. Flaring arches. III The subflare of June 27, 1980, and its related extended arch. Solar Physics, v. 134, p. 145-169, 1991.

FÜRST, E.; BENZ, A. O.; HIRTH, W. About the relation between radio and soft $\mathrm{X}$-ray emission in case of very weak solar activity. Astronomy and Astrophysics, v. 107, p. 178185, 1982.

GÜDEL, M.; BENZ, A. O. A catalogue of decimetric solar flare radio emission. Astronomy and Astrophysics Supplement Series, v. 75, p. 243-259, 1988.

GUIDICE, D. A.; CASTELLI, J. P. The use of extraterrestrial radio sources in the measurement of antenna parameters. IEEE Transactions on Aerospace and Electronic Systems, v. 7, n. 2, p. 226-234, 1971.

KARLICKÝ, M.; TLAMICHA, A. The trajectories of U-type solar radio bursts. Astronomical Institutes of Czechoslovakia Bulletin, v. 27, n. 4, p. 223-226, 1976.

MCLEAN, D. J. Metrewave solar radio bursts. In: MCLEAN, D. J.; LABRUM, N. R. (Eds.). Solar radiophysics: Studies of emission from the sun at metre wavelengths. Cambridge: Cambridge University Press, 1985. Chapter 3.

MELENDEZ-MORENO, J. L. Explosões solares decimétricas tipo III associadas com a fase impulsiva do "flare" solar. Dissertação (Mestrado em Ciência Espacial) INPE, São José dos Campos. 1997. (INPE-6382TDI/601)
MELENDEZ, J. L. et al. Statistical analysis of high-frequency decimetric type III bursts. Solar Physics, v. 187, p. 77-88, 1999.

MÉSZÁROSOVÁ, H. et al. Solar decimetric type III bursts in semi-closed magnetic field structures. Astronomy and Astrophysics, v. 484, n. 2, p. 529-536, 2008.

NING, Z. J. et al. Solar partial N-burst. Chinese Journal of Astronomy and Astrophysics, v. 3, n. 4, p. 381-390, 2003.

ROSNER, R.; TUCKER, W. H.; VIANA, G. S. Dynamics of the quiescent solar corona. The Astrophysical Journal, v. 220, p. 643-665, 1978.

SAWANT, H. S.; FERNANDES, F. C. R.; NERI, J. A. C. F. Microwave type III-RS bursts. Astrophysical Journal Supplement Series, v. 90, n. 2, p. 689-691, 1994.

SAWANT, H. S. et al. Brazilian Solar Spectroscope (BSS). Solar Physics, v. 200, n. 1-2, p. 167-176, 2001.

STONE, R. G.; FAINBERG, J. A U-Type Solar Radio Burst Originating in the Outer Corona. Solar Physics, v. 20, p. 106-111, 1971.

WANG, M. et al. Observations of Microwave Type-U Bursts. Solar Physics, v. 199, p. 157164, 2001.

WILD, J. P.; SMERD, S. F.; WEISS, A. A. Solar Bursts. Annual Review of Astronomy and Astrophysics, v. 1, p. 291, 1963.

YAO, J.-X. et al. A solar type $U$ burst in the decimetric range. Advances in Space Research, v. 20, n. 12, p. 2351-2354, 1997a.

YAO, J.-X. et al. Analysis of a solar decimetric U-type burst. Chinese Journal of Astronomy and Astrophysics, v. 21, n. 2, p. 221-228, 1997b. 